\title{
Nanoscale Mapping of Recombinant Viral Proteins: From Cells to Virus-Like Particles
}

\author{
Maria Arista-Romero, Pietro Delcanale, Silvia Pujals,* and Lorenzo Albertazzi* \\ Cite This: ACS Photonics 2022, 9, 101-109 \\ Read Online
}

ACCESS |

Шll Metrics \& More

Article Recommendations

Supporting Information

ABSTRACT: Influenza recombinant proteins and virus-like particles (VLPs) play an important role in vaccine development (e.g., CadiFlu$S)$. However, their production from mammalian cells suffers from low yields and lack of control of the final VLPs. To improve these issues, characterization techniques able to visualize and quantify the different steps of the process are needed. Fluorescence microscopy represents a powerful tool able to image multiple protein targets; however, its limited resolution hinders the study of viral constructs. Here, we propose the use of super-resolution microscopy and in particular of DNA-point accumulation for imaging in nanoscale topography (DNA-PAINT) microscopy as a characterization method for recombinant viral proteins on both cells and VLPs. We were able

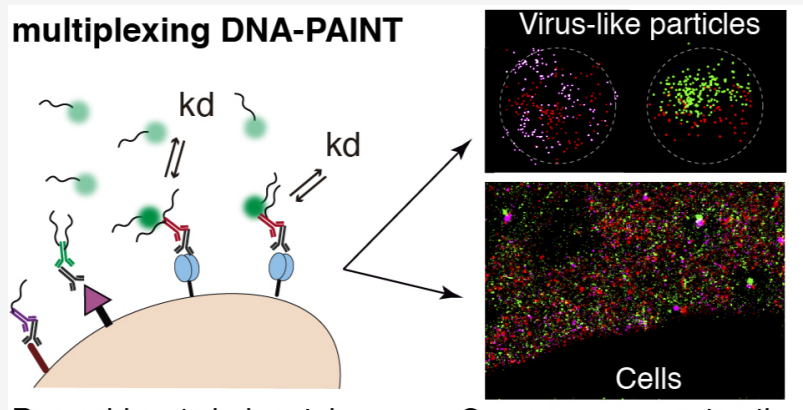

Recombinant viral proteins

Super-ress reconstruction to quantify the amount of the three main influenza proteins (hemagglutinin (HA), neuraminidase (NA), and ion channel matrix protein 2 (M2)) per cell and per VLP with nanometer resolution and single-molecule sensitivity, proving that DNA-PAINT is a powerful technique to characterize recombinant viral constructs.

KEYWORDS: super-resolution microscopy, virus-like particles, DNA-PAINT, single-particle analysis, recombinant proteins, single-molecule localization microscopy

$\mathrm{I}$ nfluenza virus-like particles (VLPs) are currently under study for their promising role as vaccines, ${ }^{1-7}$ and one VLP vaccine against influenza is on the market (CadiFlu-S, CPL Biological). ${ }^{8,9}$ They consist of small structures $(\sim 100 \mathrm{~nm}$ width) that mimic the viral particle with multicomponent organization yet without genetic material ${ }^{10,11}$ and thus are unable to replicate.

The process of the production of enveloped VLPs in mammalian cells is simple and only needs transfected cells expressing recombinant proteins of the virus on the membrane, which would then self-assemble to release empty viruses. ${ }^{12-15}$ Moreover, this process is free of contaminants (e.g., baculovirus ${ }^{2,16,17}$ ) and allows for protein post-translational modifications such as glycosylation and the incorporation of lipopolysaccharides. ${ }^{10,11,16-20}$ However, they suffer from several limitations such as low yield of the production and lack of control that often results in heterogeneous VLP production.

Therefore, significant efforts have been dedicated to modify cell lines to increase the expression yield of influenza VLPs and minimize heterogeneity. ${ }^{21-24}$

In this framework, the characterization of transfected cells and VLPs is crucial. Currently, viral constructs are characterized by DLS, ELISA, and Western blot and with electron microscopy (EM), providing information about their size and average protein content, but these methods do not allow the study of the spatial distribution and amount of protein per cell or per VLP and thus do not analyze the heterogeneity of the produced sample. Understanding recombinant protein expression at single-molecule, single-VLP, and single-cell level is important to guide the optimization of VLP synthesis.

Fluorescence microscopy is the logical method to use to map multiple proteins in VLPs and cells, thanks to its multicolor ability. However, due to the small size of influenza constructs $(\leq 100 \mathrm{~nm})$, it is impossible to characterize the protein expression and distribution on cells and VLPs with conventional fluorescent microscopy due to the diffraction limit of the light $(250 \mathrm{~nm}){ }^{25,26}$ In the past decade, superresolution microscopy and in particular single-molecule localization microscopy (SMLM) emerged to study and characterize viral structures. ${ }^{27-32}$

The SMLM technique called DNA-point accumulation for imaging in nanoscale topography (DNA-PAINT) ${ }^{33}$ has appeared as a great candidate to quantify and visualize targets

Received: July 30, 2021

Published: December 7, 2021 

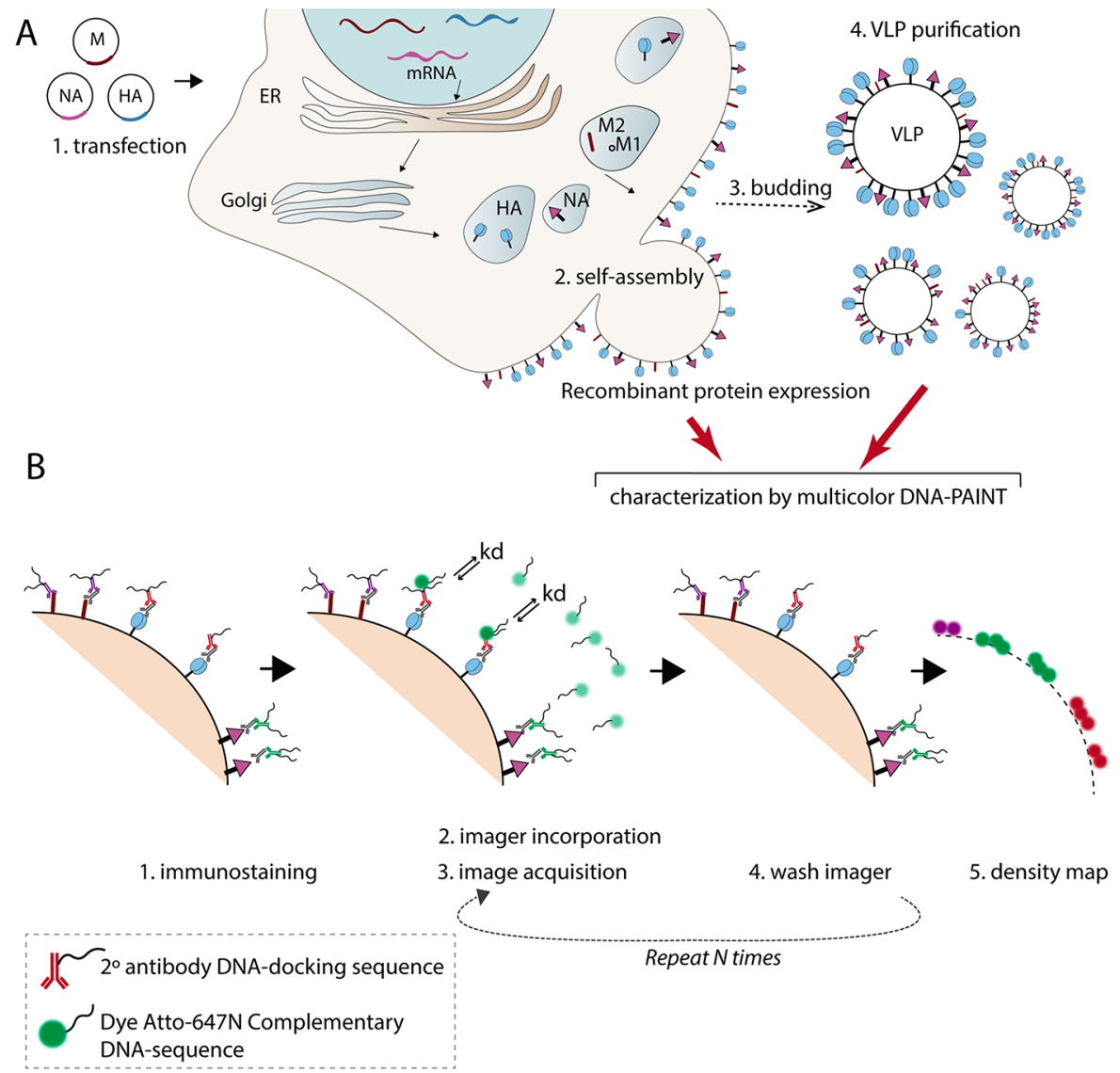

Figure 1. Diagram of the workflow followed for the characterization of recombinant viral proteins with DNA-PAINT. (A) (1) COS-7 cells were transfected with 3 different plasmids containing the 3 main envelope proteins of the influenza PR8 strain. After the transcription and translation, mature proteins reach the cell membrane. The proteins interact by self-assembly (2) and produce the virus-like particles (VLPs) by budding from the membrane (3). VLPs were then purified by ultracentrifugation with a sucrose gradient (4). (B) Both fixed cells expressing the recombinant proteins and the purified VLPs were immunostained with primary and docking-containing secondary antibodies. For DNA-PAINT imaging, the Atto-647N-conjugated imager was added to the sample. After the acquisition of the image, the imager was washed out and a second imager (with the complementary sequence for a different docking strand) was added; this process can be repeated $N$ times, and in our case, it was repeated 3 times to image the 3 target proteins sequentially. (5) After image acquisition, analysis and postprocessing of the image reveal a density map for each protein target.

in the nanoscale. ${ }^{34}$ In DNA-PAINT, single-molecule visualization is obtained by the transient and specific binding of two short sequences of DNA: one bound to the target (docking) and the other, to a dye (imager). Through the localization of these single-molecule events, a resolution of around $10 \mathrm{~nm}$ can be obtained. ${ }^{35,36}$ The use of multiple, reversible, and controlled DNA-DNA interactions allows photobleaching to be avoided, controls binding times, and permits high multiplexing imaging as well as enables up to 124 color images to be obtained and quantifies targets using quantitative PAINT (qPAINT). ${ }^{34,37,38}$ This technique has been successfully applied on nanostructures such as nanoparticles, ${ }^{39,40}$ exosomes, ${ }^{41}$ and membrane receptors ${ }^{42}$ but, up to the date, never on viral proteins.

To disclose how recombinant viral expression is carried out from mammalian cells and if it affects the final protein content of the VLPs, we characterized with DNA-PAINT the nanostructural distribution of the three main proteins of influenza (hemagglutinin, neuraminidase, and matrix protein$2)^{11}$ on recombinant cells and VLPs isolates. We quantified at single-particle level the amount of the three proteins expressed on cells, where we observed a heterogeneous production of proteins within the population. Besides, with the nanoscale mapping performed with DNA-PAINT, we studied the clustering and interactions of the viral proteins with time, while VLPs are being formed on the membrane. Finally, VLPs were imaged, quantifying the protein content and the spatial distribution of the three proteins at the single-particle level.

DNA-PAINT is an extremely powerful SMLM technique that provides solid information about recombinant viral protein expression at levels never studied.

\section{RESULTS AND DISCUSSION}

The microscopy workflow followed the steps depicted in Figure 1. First, COS-7 cells were simultaneously transfected with three different plasmids for three proteins of influenza, hemagglutinin (HA), neuraminidase (NA), and ion channel matrix protein 2 (M2) (Figure $1 \mathrm{~A}$ ), which were necessary to obtain influenza VLPs. ${ }^{11,43,44}$ Recombinant viral proteins selfassemble on the cell membrane, forming and releasing VLPs in the cell medium, where they can be collected and purified (Figure 1A). Then, cells are fixed, and VLPs were collected; multiplexed DNA-PAINT imaging was used to characterize protein expression on both VLPs and transfected cells (Figure 1B).

To do so, we preliminarily prepared secondary antibodies labeled with a DNA-docking strand, following the protocol 
A
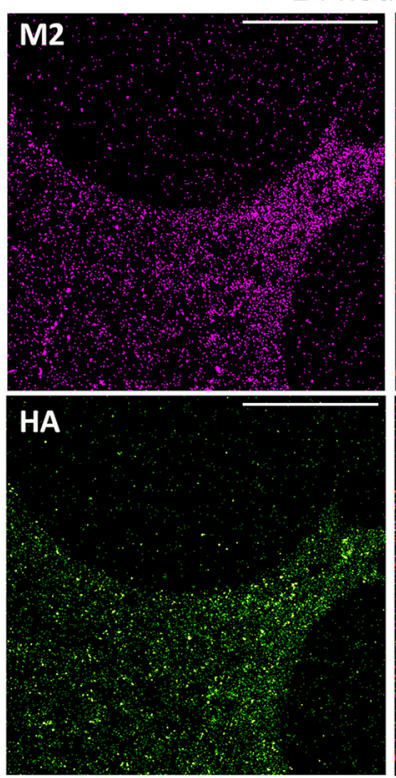

C

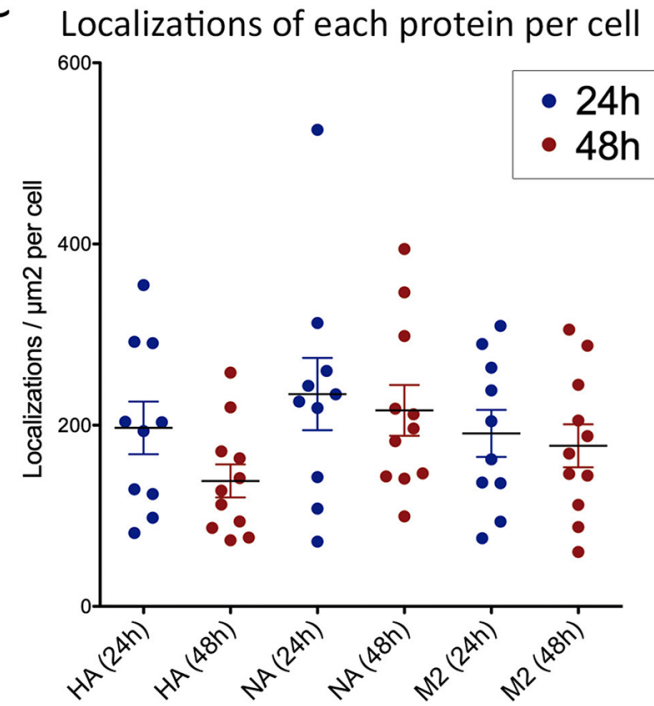

B
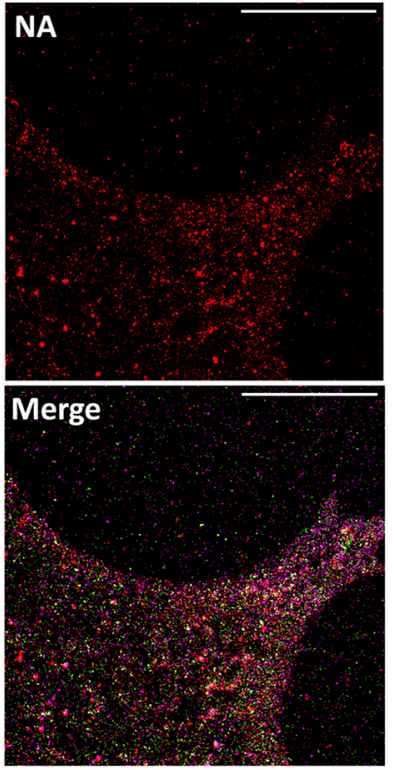

48 hours p.t.
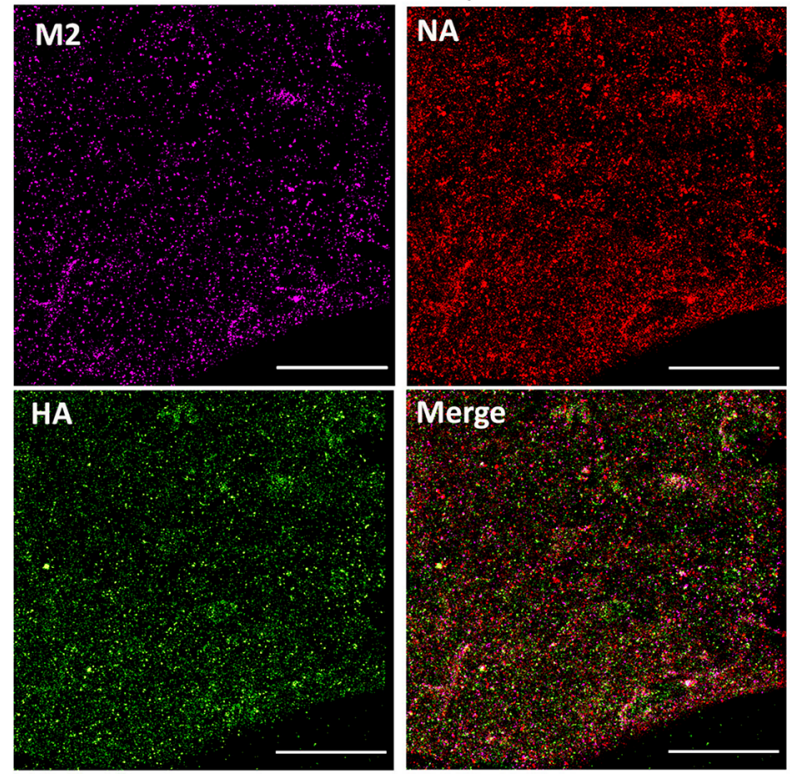

D

Viral protein localizations within the same cell

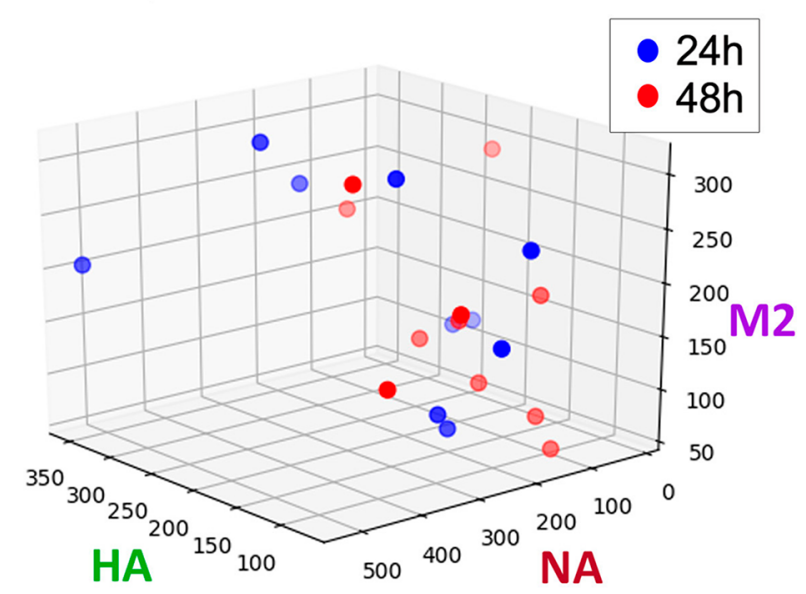

Figure 2. DNA-PAINT imaging on cells expressing recombinant proteins after 24 or $48 \mathrm{~h}$ post transfection (p.t.). DNA-PAINT super-resolution images of cell expressing the three influenza proteins (HA, NA, and M2 and the mergence of the three of them) after (A) 24 h p.t. and (B) 48 p.t. The images show an area of the cell basal membrane. Scale bar: $5 \mu \mathrm{m}$. (C) Quantification of the localization density of HA, NA, and M2 at $24 \mathrm{~h}$ and 48 h p.t. per cell $(n=10$; one point, one cell). Blue: 24 h p.t.; red: 48 h p.t. Mean and SD. (D) Viral protein localizations of the three recombinant proteins expressed within the same cell $(n=10$; one point, one cell). Blue: 24 h p.t.; red: 48 h p.t. The axes HA, NA, and M2 represent the localization density of each protein.

from Schnitzbauer et al. ${ }^{45}$ (Table S1). Each secondary antibody paired approximately 2.5 docking strains, obtaining comparable amounts of localizations (between 8 and 10 localizations per single antibody, Figure S11). We then immunostained each protein of interest (HA, M2, and NA) with a specific primary antibody and the corresponding secondary antibody, bearing a docking strand. In this way, each protein is labeled with docking strands having a specific oligonucleotide sequence.

Finally, the super-resolution DNA-PAINT images were acquired by adding the imager, a DNA strand conjugated with Atto- $647 \mathrm{~N}$, so that each imager targets the secondary antibody exposing its complementary docking strand. Since the three imagers employed in this study bear the same dye (Atto-
$647 \mathrm{~N}$ ) and only differ in the oligonucleotide sequence, they were introduced sequentially to perform multiplexed imaging of the three proteins. As illustrated in Figure 1B, the first DNAPAINT image of a target protein is acquired upon introduction of the corresponding imager in solution; subsequently, the solution is exchanged and replaced with one containing another imager, so that a different target protein is imaged with DNA-PAINT. The process is repeated until DNA-PAINT images for each target protein are acquired. A chamber-tubing system connected to a pump was used to exchange solutions while the sample was maintained in the same position (Figure S1). Finally, a false color was assigned to each sequentially acquired DNA-PAINT image, and the images were merged to 
A
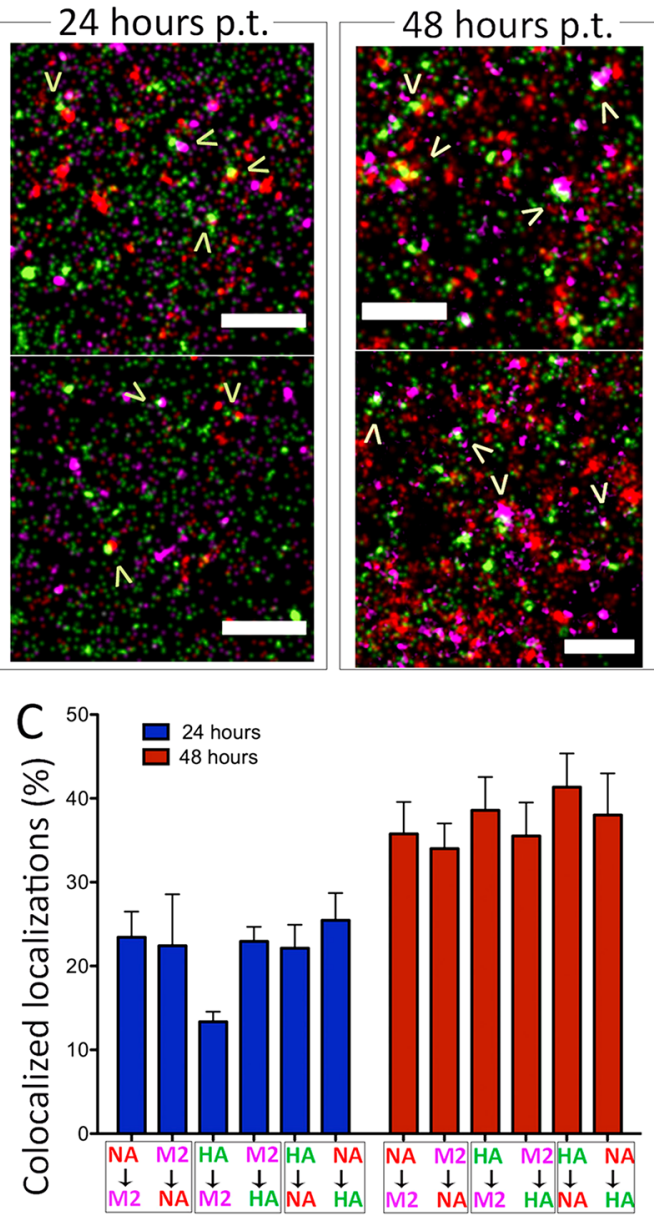

Pairs of proteins compared

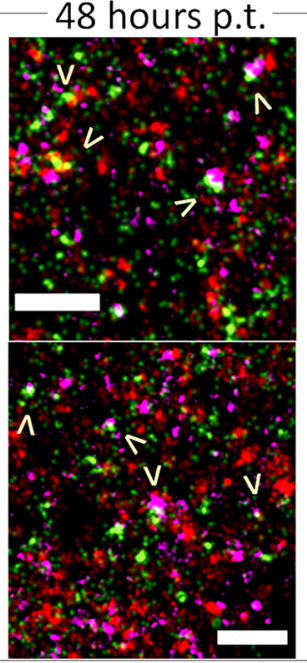

B
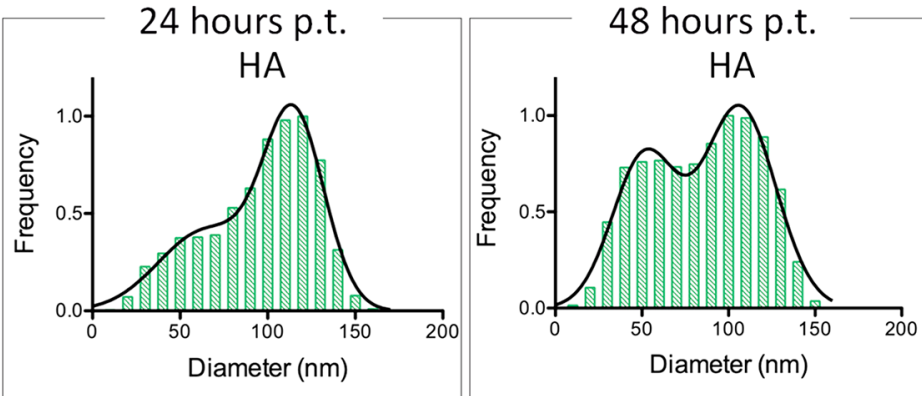

NA
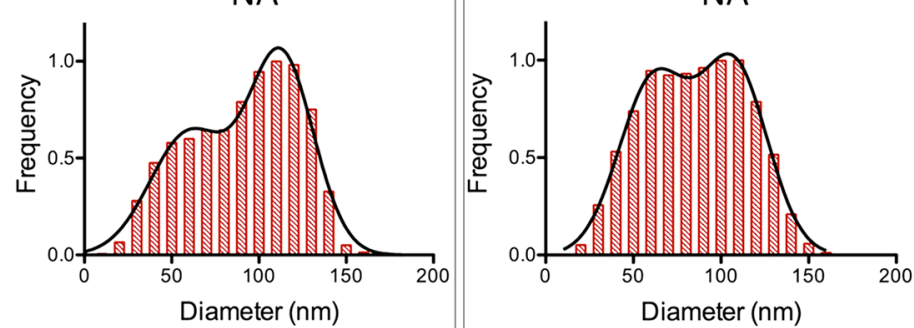

$\mathrm{M} 2$

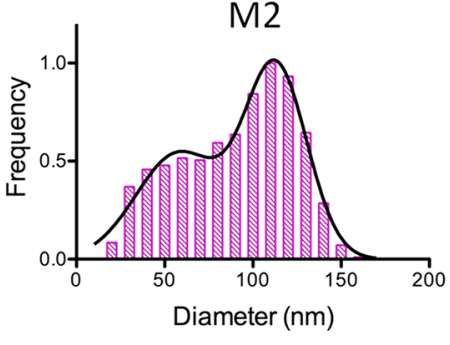

2 way-ANOVA (Time) $\mathrm{P}<0.0001$

Figure 3. Recombinant protein distribution along the membrane of cells at 24 and 48 h post-transfection (p.t.). (A) Close-up of DNA-PAINT super-resolution images of the 3 proteins expressed within the same cell at 24 and 48 h p.t. The arrowheads indicate the bigger cluster agglomerations and colocalizations. Scale bar, $500 \mathrm{~nm}$. Red: NA; magenta: M2; green: HA. (B) Normalized cluster size distribution of each protein expressed in the whole population of cells at 24 and $48 \mathrm{~h}$ p.t. The normalized distribution of the diameter (nm) showed two populations where black lines show the result of a fitting with a two-Gaussian model number of clusters measured, $n=6000$. (C) Percentage of colocalizations of the localizations of the three pairs of proteins within the same cell $(n=3)$. 2 -way ANOVA test on the factor "time", $P<0.0001$; colocalizations at 24 and 48 h p.t. are statistically significantly different.

reconstruct a final multicolor DNA-PAINT image of the three target proteins.

To identify the timeline of the post-transfection (p.t.) expression of recombinant proteins, we first checked the expression after 24 and 48 h p.t. with fluorescence microscopy (Figure S2). Results confirmed that after $24 \mathrm{~h}$ the expression levels were similar to those at $48 \mathrm{~h}$ p.t., which is the time point where VLPs are harvested from the culture medium. ${ }^{14,18}$ Thus, to quantitatively monitor the recombinant protein expression, DNA-PAINT images of the three proteins on the cell membrane were acquired at both 24 and 48 h p.t., using the same imager concentration $(1.5 \mathrm{nM})$ to obtain comparable results.

Figure 2A,B shows representative super-resolution images obtained for each protein (M2, HA, NA) in the same region of the membrane. Visually, the expression level of the three proteins seemed similar at both 24 h p.t. (Figure $2 \mathrm{~A}$ ) and $48 \mathrm{~h}$ p.t. (Figure 2B). In order to quantitatively compare expression levels for the different proteins, in each image, the density of the DNA-PAINT localizations was calculated within an area corresponding to the cell.

Figure 2C reports the measured values of localization density (localizations $/ \mu \mathrm{m}^{2}$ ) for the different proteins (HA, NA, $\mathrm{M} 2$ ) at 24 and $48 \mathrm{~h}$ p.t., obtained from the analysis of multiple cells. Figure 2D reports complementary information where each point in the three-dimensional plot represents a cell, and the three coordinates of the point are the measured localization densities of the three proteins (HA, NA, M2).

When we examined the localization densities in the whole population of cells analyzed (Figure $2 \mathrm{C}$ ), we observed that the average values obtained for the three proteins were similar. The densities ranged between 100 and 400 localizations $/ \mu \mathrm{m}^{2}$, independently of the protein type. Moreover, no significant difference was observed between 24 and 48 h p.t, consistent with the qualitative measurement obtained with fluorescence microscopy (Figure S2).

However, this data also provides a quantification of cell-tocell differences in protein expression levels. Despite cells being 
transfected at the same time and treated in the same way, Figure 2C shows a relevant heterogeneity in the measured density between single cells. Though the average values displayed are comparable, single cells produced recombinant proteins at different yields, pointing out the heterogeneity of this recombinant expression system. This has deep implications for the production of VLPs as different cells will produce VLPs with different protein contents.

Finally, thanks to the multiplexing ability of DNA-PAINT, we could relate and compare the localization density of the three proteins expressed within the same cell (Figure 2D). No clear correlation is observed between localization densities of the three proteins within the same cell. This suggests that the expression of each protein is independent of the other. Importantly, control experiments performed with other recombinant proteins (LacZ) and noncomplementary imagers (Figure S3) yielded significantly lower localization densities, ensuring that nonspecific signals from unwanted interactions are negligible.

Overall, these quantitative results show that COS-7 cells expressed the three recombinant proteins with similar levels, on average, but with a high cell-to-cell variability. Remarkably, this information is not achieved with traditional bulk techniques such as ELISA or Western blot. Additionally, no correlation is observed between expression levels of the three recombinant proteins on the same cell. These findings highlight that a constant and reproducible expression of recombinant proteins is hardly achieved at the level of a single cell.

Moreover, observing a close-up of the merged areas on the super-resolution images (Figure 3A), we could detect that a fraction of proteins was arranged in clusters (arrowheads), a possible indication of VLP budding formation. ${ }^{12}$ In order to measure the observed differences in protein arrangement with time, we carried out an analysis of cluster size and composition (see the methods in the Supporting Information).

At $24 \mathrm{~h}$ p.t., the normalized diameter distribution (Figure $3 \mathrm{~B}$, left) of the identified clusters displayed two main cluster populations for all proteins: a smaller population with a peak value at $\sim 60 \mathrm{~nm}$ diameter and a main population with a peak value at $\sim 110 \mathrm{~nm}$. The relative amplitude of the two populations is similar for the three proteins: the peak at $\sim 110 \mathrm{~nm}$ diameter is roughly two times higher that the peak at $\sim 60 \mathrm{~nm}$ diameter. A more precise comparison is obtained by fitting the distributions with a two-Gaussian model and calculating the peak ratio (amplitude of peak at $\sim 110 \mathrm{~nm} /$ amplitude of peak at $\sim 60 \mathrm{~nm}$ ): 2.4 for HA, 1.6 for NA, and 1.8 for M2. In addition, discerning the two populations closely (Figures S4 and S5), we observed that $\sim 75 \%$ of the clusters found presented a sized distribution between 71 and $170 \mathrm{~nm}$, whereas the presence of small clusters is a minority.

Notably, the distributions changed significantly at 48 h p.t. (Figure 3B, right; Figure S4). While the positions of the peaks (at $\sim 60$ and $\sim 110 \mathrm{~nm}$ ) are very similar to those observed at 24 $\mathrm{h}$ p.t., their relative amplitude is much more alike if compared with the previous case. The measured peak ratios obtained at $48 \mathrm{~h}$ p.t. are 1.3 for $\mathrm{HA}, 1.1$ for NA, and 1.7 for M2. In particular, clusters of NA proteins even show two populations with the same amplitude (Figures $3 \mathrm{~B}$ and S4). We hypothesized that this moderate change in cluster distribution could be related to the VLP release: while small clusters remain constant in time (from 24 to 48 h p.t.), the big aggregation of proteins is more likely to form VLPs and be released at $48 \mathrm{~h}$ p.t than at $24 \mathrm{~h}$ p.t.

To confirm this concept, we measured colocalization between all the proteins (Figure 3C) with a previously developed analysis tool, Clus-DoC, by Pageon et al. ${ }^{46}$ Briefly, this algorithm compares the local density of localizations of two channels, i.e., two proteins. For each localization of a DNA-PAINT image, the surrounding localization density in both channels is calculated within an area of increasing radius, providing the density gradients of both channels. These gradients are then compared to yield a degree of colocalization (DoC) parameter, ranging from -1 (full segregation) to +1 (full colocalization), which is assigned to each localization. A representative output of the analysis is given in Figure S6A. We then selected a threshold value of 0.4 for the DoC, above which a localization significantly colocalizes with surrounding localizations of the other channel. This choice is based on a previous observation that the $\mathrm{DoC}$ values obtained for two identical images shifted by $10 \mathrm{~nm}$ (i.e., the highest resolution achieved by PAINT) were above 0.4 .

Figure 3C summarizes the colocalization results for each protein pair obtained at 24 and 48 h p.t., where the percentage of colocalized localizations is the percentage of localization having a DoC above 0.4 . The general percentage of colocalized proteins at $24 \mathrm{~h}$ oscillated between $12 \%$ and $22 \%$, except for HA compared to M2, which exhibited an unexpected low level of colocalization (Figure S6B). However, at $48 \mathrm{~h}$, the colocalization boosted up to $\sim 40 \%$ for all protein pairs. This increase protein colocalization at $48 \mathrm{~h}$ p.t. was statistically significant and correlates with the decreased amount of large clusters of proteins, as highlighted in Figure 3B. These facts are consistent with an enhanced production and release of VLPs with a width of around $100 \mathrm{~nm}$ occurring after 48 h p.t. with respect to $24 \mathrm{~h}$.

In brief, our method allows the imaging and localization of recombinant viral proteins on the cell membrane at the nanoscale, not only retrieving quantitative information about the amount of protein produced but also correlating the extent of expression for each protein at the single-cell level. Further analysis allows the quantification of the clusters formed on the membrane and of the interaction of the three proteins, opening the possibility to describe the evolution and colocalization of recombinant proteins on the membrane with time.

Having measured the heterogeneous expression in cells and how proteins organize with respect to time on the cell membrane prior to the release of VLP, we focused our attention on the protein mapping within isolated VLPs using multiplexed DNA-PAINT. By quantifying the amount of the three proteins per single VLP at single-particle level, we aimed to relate the previously studied protein expression at cellular level with the resulting protein content within single VLPs stemming from the cells.

Multiplexed DNA-PAINT characterization of nanostructures has been deeply described on nanoparticles ${ }^{39}$ and exosomes, $^{41}$ which have similar size and shape to influenza VLPs. For this purpose, we produced, isolated, and labeled VLPs produced from COS-7 cells previously studied and applied DNA-PAINT imaging to describe the distribution of the recombinant proteins at the single-particle level. To make sure the VLPs were properly isolated, all the fractions recovered from the ultracentrifugation sucrose gradient (Figure S7) were measured with dynamic light scattering (DLS) to select the samples with low polydispersity (PDI) and the right 

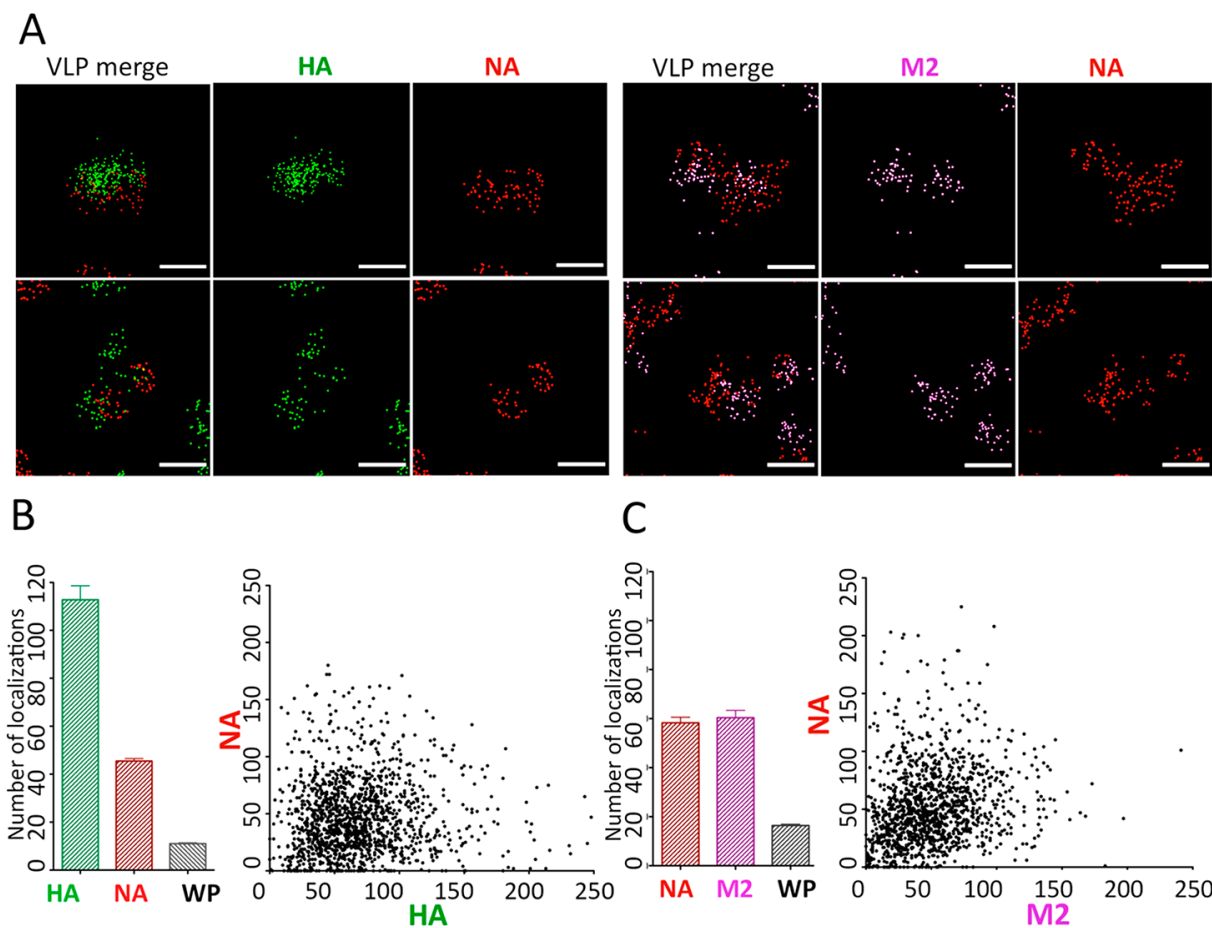

\section{C}
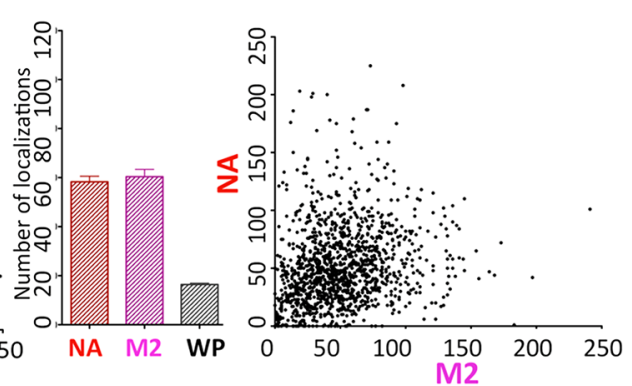

Figure 4. DNA-PAINT characterization of recombinant viral proteins on the resulting VLPs obtained at $48 \mathrm{~h}$ post-transfection. (A) DNA-PAINT super-resolution images of the two samples prepared: a single VLP immunostained against HA and NA (left) and M2 and NA (right). Scale bar $100 \mathrm{~nm} . N=1400$. (B) Left: Average number of the localizations of HA and NA per VLP. WP represents the negative control obtained with the wrong pairing (WP) of docking and imager, i.e., imager mispairing on VLP (mean and SEM). Right: localizations of NA and HA within the same VLP. (C) Left: Average of the localizations of M2 and NA per VLP. WP represents the negative control or wrong pairing (WP); imager mispairing on VLP (mean and SEM). Right: localizations of NA and M2 within the same VLP. $N=1300$.

size $(\sim 100 \mathrm{~nm})$ (fraction corresponding to $42-45 \%$ sucrose). Besides DLS, the size of the recovered VLPs was determined with conventional techniques such as transmission electron microscopy (TEM) and super-resolution microscopy (STORM), which measured between 75 and $110 \mathrm{~nm}$ (Figure S8).

To perform the multiplexed DNA-PAINT, VLPs were first labeled with a hydrophobic dye (DiI) that was employed as a reference to identify the particles on the glass coverslip. The immunostaining of target proteins on VLPs (HA, NA, M2) was performed in solution, using ultracentrifugation for removing the unbound antibodies. In this experiment, we performed a two-label immunostaining (details in the methods in the Supporting Information); hence, we divided the protein identification in two pairs of proteins: we first labeled HA and NA and then M2 and NA, performing 2 separate experiments with the same conditions. Antibody labeled VLPs were incubated on a clean glass-bottom Petri dish for $30 \mathrm{~min}$, followed by adding the first imager for the acquisition of a DNA-PAINT image, washing, and adding the second imager with the previously described tubing system (Figure S1).

During the image acquisition, DiI was used as a drifting corrector and further to localize the VLPs and analyze the localizations of each protein around them, measuring the total amount of localization surrounding each DiI signal (details in the methods in the Supporting Information).

Figure $4 \mathrm{~A}$ shows the reconstructed images obtained for the two immunostained proteins on VLP. The nanoscale distribution of the localizations from HA (green) and NA (red) within the same VLP was directly visualized with DNAPAINT. It can be observed that both HA and NA localizations formed small clusters along the structure in some cases (e.g., lower panels), while being more evenly distributed in other cases (e.g., upper panels). Generally, the population of VLPs displayed a strong heterogeneity, both intraparticle and interparticle, within the population of VLPs studied. The average value of the total amount of localization per VLP for both proteins is displayed in Figure 4B, where HA showed an average of $\sim 120$ localizations per VLP while NA had $\sim 56$ localizations. When we plotted the distribution of the localizations in the whole population of VLPs measured (Figure S9), we observed that HA displays a broader distribution than NA.

Furthermore, since we could measure the amount of localizations at single-particle level, we could match the amount of both proteins within the same VLP (Figure 4B, right plot). We observe an essential lack of correlation between the amounts of localizations of NA and HA. Indeed, no clear trend can be identified in this plot, which simply points out that each VLP contained a different amount of proteins, confirming the enormous heterogeneity of the recombinant protein presence in the population of VLPs.

Similarly, the nanoscale distribution of M2 and NA proteins (magenta and red, respectively) exhibited marked heterogeneity, both intraparticle and interparticle (Figure 4A-C). Interestingly, NA and M2 proteins generated a nearly identical amount of localizations on the surface of analyzed VLPs, both containing $\sim 60$ localizations (Figures $4 \mathrm{C}$ and S9). In this case, when the presence of both proteins within the same VLP particle was compared (Figure 4C, right), a lack of clear correlation of the incidence of both proteins could be seen. To check the specificity of the imager-docking recognition, we performed two control experiments using imagers with a DNA sequence that was noncomplementary to that of the docking 
(wrong pairing WP) (Figure S10), obtaining a significantly lower amount of localizations, proving the robustness and specificity of the imaging DNA-PAINT system.

Surprisingly, the relative amount of localizations between the different proteins on the VLPs is not aligned with the amount of localizations expressed on the cell membranes. In VLPs, HA is doubly expressed, while in cells, its expression is similar to the other proteins. This could be explained by the selfassembly nature of the VLP formation, where HA has a higher propensity to be incorporated. All in all, with DNA-PAINT, it was possible to characterize the allocation of the recombinant proteins at single-particle level, observing their intraparticle and interparticle distribution and quantifying the amount of localizations of both proteins within the same VLP, proving the heterogeneity in protein expression and in protein content for three proteins in the total population of VLPs.

\section{CONCLUSIONS}

In conclusion, DNA-PAINT proved to be a robust, sensitive, and versatile tool to characterize the recombinant viral protein expression. Thanks to the multiplexing ability of DNA-PAINT, we could quantify the expression of three different recombinant proteins on biological samples using exactly the same imaging conditions (laser power, dye, and optics/filters).

First, we successfully characterized the heterogeneous expression of the three proteins of influenza transfected at the same time on COS-7 cells, comparing the expression on the same surface area with time. The results confirmed that recombinant proteins are expressed heterogeneously in mammalian cells with large cell-to-cell variability in the level of the three proteins.

Thus, we could characterize the distribution of the proteins that were organized in clusters on the membrane, achieving resolutions of $10-15 \mathrm{~nm}$ that allowed the identification of two populations of clusters, small $(60-70 \mathrm{~nm})$ and big (100-110 $\mathrm{nm})$, in different proportions that changed with time. Concurrently, the localizations retrieved in the superresolution analysis allowed a colocalization analysis, showing that with time the clusters increased the percentage of colocalization. All the information obtained would help to describe the recombinant expression model, and even though the expression of the proteins was quite heterogeneous, the protein distribution and interaction stayed balanced; most of the proteins displayed similar sizes and percentages of colocalizations that changed evenly in time.

Furthermore, DNA-PAINT provided single-particle insights of the recombinant proteins on VLPs formed from COS-7 cells, allowing the quantification and visualization of the distribution of the proteins along the particle, offering singleparticle measurements of the two proteins characterized at the VLP level. Remarkably, NA and M2 presented similar features in terms of the amount of localizations and cluster distribution along the particle, while HA was doubly expressed. These localizations per VLP were not correlated to the one observed in the cells, indicating that the VLP content is not related to the total amount of protein expressed on the cells but to the viral budding mechanism. The visualization of where the proteins are distributed on the VLP at the single-particle level would lead us to understand the heterogeneity of the population of intraparticle and interparticle VLPs, a critical feature for clinical approaches.

This nanocharacterization and semiquantification based on DNA-PAINT is a powerful tool to study and define new vaccine systems. In fact, this insight could be key in the characterization of new vaccines, such as COVID-19 vaccines, consisting of mRNA vectors that produce recombinant proteins in mammalian cells. ${ }^{47-49}$

\section{ASSOCIATED CONTENT}

\section{(s) Supporting Information}

The Supporting Information is available free of charge at https://pubs.acs.org/doi/10.1021/acsphotonics.1c01154.

Setup of the chamber for imaging multicolor DNAPAINT on the microscope; fluorescence microscopy images and mean intensity of fluorescence; density of DNA-PAINT localizations; cluster size and frequency distributions; percentage of the populations of clusters; output of the Clus-DoC analysis; DLS measurements; measurements of the size of VLPs, dynamic light scattering (DLS) measurements, TEM and STORM images; DNA-PAINT of VLPs; materials and methods; list of oligos (docking-imagers); analysis description (PDF)

\section{AUTHOR INFORMATION}

\section{Corresponding Authors}

Lorenzo Albertazzi - Nanoscopy for Nanomedicine Group, Institute for Bioengineering of Catalonia (IBEC), The Barcelona Institute of Science and Technology, 08028 Barcelona, Spain; Department of Biomedical Engineering, Institute for Complex Molecular Systems (ICMS), Eindhoven University of Technology, 5612AZ Eindhoven, The Netherlands; Email: 1.albertazzi@tue.nl

Silvia Pujals - Nanoscopy for Nanomedicine Group, Institute for Bioengineering of Catalonia (IBEC), The Barcelona Institute of Science and Technology, 08028 Barcelona, Spain; Email: spujals@ibecbarcelona.eu

\section{Authors}

Maria Arista-Romero - Nanoscopy for Nanomedicine Group, Institute for Bioengineering of Catalonia (IBEC), The Barcelona Institute of Science and Technology, 08028 Barcelona, Spain

Pietro Delcanale - Dipartimento di Scienze Matematiche, Fisiche e Informatiche, Università di Parma, 43124 Parma, Italy; orcid.org/0000-0001-8235-765X

Complete contact information is available at:

https://pubs.acs.org/10.1021/acsphotonics.1c01154

\section{Author Contributions}

M.A-R prepared all the protocols and samples, acquired the super-resolution images, and carried out the analysis. P.D. developed the cluster algorithms and analysis codes. S.P. and L.A. supervised all the work. M.A-R wrote the paper, and all authors have given approval to the final version of the manuscript.

\section{Notes}

The authors declare no competing financial interest.

\section{ACKNOWLEDGMENTS}

The authors would like to acknowledge the groups of Dr. Nico Callewaert and Dr. Xavier Saelens from VIB-UGENT Center for Medical Biotechnology for providing the influenza plasmids used in this paper (PHW2000 HA, NA, and M). M.A.-R. is grateful to the Spanish Ministry of Universities and the 
European Social Fund for the funding of his research activity by the research project (FPI16-SEV-2014-0425-16-1) (Ayudas para contratos predoctorales para la formación de doctores 2016). S.P. and L.A acknowledge the financial support by the Spanish Ministry of Science and Innovation (PID2019109450RB-I00/AEI/10.13039/501100011033), European Research Council/Horizon 2020 (ERC-StG-757397), "la Caixa" Foundation (ID 100010434), and the Generalitat de Catalunya (through the CERCA program and 2017 SGR 01536).

\section{REFERENCES}

(1) Kang, S. M.; Pushko, P.; Bright, R. A.; Smith, G.; Compans, R. W. Influenza Virus-Like Particles as Pandemic Vaccines. In Vaccines for Pandemic Influenza; Current Topics in Microbiology and Immunology; Springer: Berlin, Heidelberg, 2009; pp 269-289; DOI: $10.1007 / 978-3-540-92165-314$.

(2) Fuenmayor, J.; Gòdia, F.; Cervera, L. Production of Virus-like Particles for Vaccines. New Biotechnol. 2017, 39 (Pt B), 174-180.

(3) Kang, S.-M.; Kim, M.-C.; Compans, R. W. Virus-like Particles as Universal Influenza Vaccines. Expert Rev. Vaccines 2012, 11 (8), 9951007.

(4) Wang, Y.; Deng, L.; Kang, S.-M.; Wang, B.-Z. Universal Influenza Vaccines: From Viruses to Nanoparticles. Expert Rev. Vaccines 2018, 17 (11), 967-976.

(5) Haynes, J. R. Influenza Virus-like Particle Vaccines. Expert Rev. Vaccines 2009, 8 (4), 435-445.

(6) Kang, H.-J.; Chu, K.-B.; Lee, D.-H.; Lee, S.-H.; Park, B. R.; Kim, M.-C.; Kang, S.-M.; Quan, F.-S. Influenza M2 Virus-like Particle Vaccination Enhances Protection in Combination with Avian Influenza HA VLPs. PLoS One 2019, 14 (6), No. e0216871.

(7) Kim, K.-H.; Kwon, Y.-M.; Lee, Y.-T.; Kim, M.-C.; Hwang, H. S.; Ko, E.-J.; Lee, Y.; Choi, H.-J.; Kang, S.-M. Virus-Like Particles Are a Superior Platform for Presenting M2e Epitopes to Prime Humoral and Cellular Immunity against Influenza Virus. Vaccines (Basel, Switz.) 2018, 6 (4), 66.

(8) Sparrow, E.; Wood, J. G.; Chadwick, C.; Newall, A. T.; Torvaldsen, S.; Moen, A.; Torelli, G. Global Production Capacity of Seasonal and Pandemic Influenza Vaccines in 2019. Vaccine 2021, 39 (3), 512-520.

(9) Press Release; CPL Biologicals Launches Cadiflu-S, World's First Virus like Particle (VLP) Vaccine for Seasonal Influenza; http://cplbio. com/press-post/november-17-2016/.

(10) McCraw, D. M.; Gallagher, J. R.; Torian, U.; Myers, M. L.; Conlon, M. T.; Gulati, N. M.; Harris, A. K. Structural Analysis of Influenza Vaccine Virus-like Particles Reveals a Multicomponent Organization. Sci. Rep. 2018, 8 (1), 1-16.

(11) Wu, C.-Y.; Yeh, Y.-C.; Yang, Y.-C.; Chou, C.; Liu, M.-T.; Wu, H.-S.; Chan, J.-T.; Hsiao, P.-W. Mammalian Expression of Virus-Like Particles for Advanced Mimicry of Authentic Influenza Virus. PLoS One 2010, 5 (3), No. e9784.

(12) Nayak, D. P.; Balogun, R. A.; Yamada, H.; Zhou, Z. H.; Barman, S. Influenza Virus Morphogenesis and Budding. Virus Res. 2009, 143 (2), 147-161.

(13) Latham, T.; Galarza, J. M. Formation of Wild-Type and Chimeric Influenza Virus-Like Particles Following Simultaneous Expression of Only Four Structural Proteins. J. Virol. 2001, 75 (13), 6154-6165.

(14) Chen, B. J.; Leser, G. P.; Morita, E.; Lamb, R. A. Influenza Virus Hemagglutinin and Neuraminidase, but Not the Matrix Protein, Are Required for Assembly and Budding of Plasmid-Derived VirusLike Particles. J. Virol. 2007, 81 (13), 7111-7123.

(15) Durous, L.; Rosa-Calatrava, M.; Petiot, E. Advances in Influenza Virus-like Particles Bioprocesses. Expert Rev. Vaccines 2019, 18 (12), 1285-1300.

(16) Dai, S.; Wang, H.; Deng, F. Advances and Challenges in Enveloped Virus-like Particle (VLP)-Based Vaccines. Journal of Immunological Sciences 2018, 2 (2), 36.
(17) Nooraei, S.; Bahrulolum, H.; Hoseini, Z. S.; Katalani, C.; Hajizade, A.; Easton, A. J.; Ahmadian, G. Virus-like Particles: Preparation, Immunogenicity and Their Roles as Nanovaccines and Drug Nanocarriers. J. Nanobiotechnol. 2021, 19 (1), 59.

(18) Thompson, C. M.; Petiot, E.; Mullick, A.; Aucoin, M. G.; Henry, O.; Kamen, A. A. Critical Assessment of Influenza VLP Production in Sf9 and HEK293 Expression Systems. BMC Biotechnol. 2015, 15 (1), 31.

(19) Pushko, P.; Tretyakova, I. Influenza Virus Like Particles (VLPs): Opportunities for H7N9 Vaccine Development. Viruses 2020, 12 (5), 518.

(20) Sarkar, B.; Islam, S. S.; Zohora, U. S.; Ullah, M. A. Virus like Particles- A Recent Advancement in Vaccine Development. Korean J. Microbiol. 2019, 55 (4), 327-343.

(21) Buffin, S.; Peubez, I.; Barrière, F.; Nicolaï, M.-C.; Tapia, T.; Dhir, V.; Forma, E.; Sève, N.; Legastelois, I. Influenza A and B Viruslike Particles Produced in Mammalian Cells Are Highly Immunogenic and Induce Functional Antibodies. Vaccine 2019, 37 (46), 68576867.

(22) Venereo-Sanchez, A.; Gilbert, R.; Simoneau, M.; Caron, A.; Chahal, P.; Chen, W.; Ansorge, S.; Li, X.; Henry, O.; Kamen, A. Hemagglutinin and Neuraminidase Containing Virus-like Particles Produced in HEK-293 Suspension Culture: An Effective Influenza Vaccine Candidate. Vaccine 2016, 34 (29), 3371-3380.

(23) Venereo-Sánchez, A.; Fulton, K.; Koczka, K.; Twine, S.; Chahal, P.; Ansorge, S.; Gilbert, R.; Henry, O.; Kamen, A. Characterization of Influenza H1N1 Gag Virus-like Particles and Extracellular Vesicles Co-Produced in HEK-293SF. Vaccine 2019, 37 (47), 7100-7107.

(24) Venereo-Sanchez, A.; Simoneau, M.; Lanthier, S.; Chahal, P.; Bourget, L.; Ansorge, S.; Gilbert, R.; Henry, O.; Kamen, A. Process Intensification for High Yield Production of Influenza H1N1 Gag Virus-like Particles Using an Inducible HEK-293 Stable Cell Line. Vaccine 2017, 35 (33), 4220-4228.

(25) Abbe, E. Beiträge zur Theorie des Mikroskops und der mikroskopischen Wahrnehmung. Archiv f. mikrosk. Anatomie 1873, 9 (1), 413-468.

(26) Masters, B. R. Abbe Theory of Image Formation and Diffraction of Light in Transmitted Light Microscopes 2006, 37-49.

(27) Arista-Romero, M.; Pujals, S.; Albertazzi, L. Towards a Quantitative Single Particle Characterization by Super Resolution Microscopy: From Virus Structures to Antivirals Design. Front. Bioeng. Biotechnol. 2021, 9, 9.

(28) Laine, R. F.; Albecka, A.; van de Linde, S.; Rees, E. J.; Crump, C. M.; Kaminski, C. F. Structural Analysis of Herpes Simplex Virus by Optical Super-Resolution Imaging. Nat. Commun. 2015, 6 (1), 5980.

(29) Dahmane, S.; Doucet, C.; Le Gall, A.; Chamontin, C.; Dosset, P.; Murcy, F.; Fernandez, L.; Salas, D.; Rubinstein, E.; Mougel, M.; Nollmann, M.; Milhiet, P.-E. Nanoscale Organization of Tetraspanins during HIV-1 Budding by Correlative DSTORM/AFM. Nanoscale 2019, 11 (13), 6036-6044.

(30) Kolpe, A.; Arista-Romero, M.; Schepens, B.; Pujals, S.; Saelens, X.; Albertazzi, L. Super-Resolution Microscopy Reveals Significant Impact of M2e-Specific Monoclonal Antibodies on Influenza A Virus Filament Formation at the Host Cell Surface. Sci. Rep. 2019, 9, 9.

(31) Muranyi, W.; Malkusch, S.; Müller, B.; Heilemann, M.; Kräusslich, H.-G. Super-Resolution Microscopy Reveals Specific Recruitment of HIV-1 Envelope Proteins to Viral Assembly Sites Dependent on the Envelope C-Terminal Tail. PLoS Pathog. 2013, 9 (2), No. e1003198.

(32) Sieben, C.; Sezgin, E.; Eggeling, C.; Manley, S. Influenza A Viruses Use Multivalent Sialic Acid Clusters for Cell Binding and Receptor Activation. PLoS Pathog. 2020, 16, e1008656.

(33) Jungmann, R.; Steinhauer, C.; Scheible, M.; Kuzyk, A.; Tinnefeld, P.; Simmel, F. C. Single-Molecule Kinetics and SuperResolution Microscopy by Fluorescence Imaging of Transient Binding on DNA Origami. Nano Lett. 2010, 10 (11), 4756-4761.

(34) Jungmann, R.; Avendaño, M. S.; Dai, M.; Woehrstein, J. B.; Agasti, S. S.; Feiger, Z.; Rodal, A.; Yin, P. Quantitative Super- 
Resolution Imaging with QPAINT. Nat. Methods 2016, 13 (5), 439442.

(35) Dai, M.; Jungmann, R.; Yin, P. Optical Imaging of Individual Biomolecules in Densely Packed Clusters. Nat. Nanotechnol. 2016, 11 (9), 798-807.

(36) Jungmann, R.; Avendaño, M. S.; Woehrstein, J. B.; Dai, M.; Shih, W. M.; Yin, P. Multiplexed 3D Cellular Super-Resolution Imaging with DNA-PAINT and Exchange-PAINT. Nat. Methods 2014, 11 (3), 313-318.

(37) Agasti, S. S.; Wang, Y.; Schueder, F.; Sukumar, A.; Jungmann, R.; Yin, P. DNA-Barcoded Labeling Probes for Highly Multiplexed Exchange-PAINT Imaging. Chem. Sci. 2017, 8, 3080.

(38) Wade, O. K.; Woehrstein, J. B.; Nickels, P. C.; Strauss, S.; Stehr, F.; Stein, J.; Schueder, F.; Strauss, M. T.; Ganji, M.; Schnitzbauer, J.; Grabmayr, H.; Yin, P.; Schwille, P.; Jungmann, R. 124-Color SuperResolution Imaging by Engineering DNA-PAINT Blinking Kinetics. Nano Lett. 2019, 19 (4), 2641-2646.

(39) Delcanale, P.; Miret-Ontiveros, B.; Arista-Romero, M.; Pujals, S.; Albertazzi, L. Nanoscale Mapping Functional Sites on Nanoparticles by Points Accumulation for Imaging in Nanoscale Topography (PAINT). ACS Nano 2018, 12 (8), 7629-7637.

(40) Delcanale, P.; Albertazzi, L. DNA-PAINT Super-Resolution Imaging Data of Surface Exposed Active Sites on Particles. Data in Brief 2020, 30, 105468.

(41) Chen, C.; Zong, S.; Liu, Y.; Wang, Z.; Zhang, Y.; Chen, B.; Cui, Y. Profiling of Exosomal Biomarkers for Accurate Cancer Identification: Combining DNA-PAINT with Machine- Learning-Based Classification. Small 2019, 15 (43), 1901014.

(42) Cremers, G. A. O.; Rosier, B. J. H. M.; Riera Brillas, R.; Albertazzi, L.; de Greef, T. F. A. Efficient Small-Scale Conjugation of DNA to Primary Antibodies for Multiplexed Cellular Targeting. Bioconjugate Chem. 2019, 30 (9), 2384-2392.

(43) Pan, Y.-S.; Wei, H.-J.; Chang, C.-C.; Lin, C.-H.; Wei, T.-S.; Wu, S.-C.; Chang, D.-K. Construction and Characterization of Insect CellDerived Influenza VLP: Cell Binding, Fusion, and EGFP Incorporation. BioMed Res. Int. 2010, 2010, 506363.

(44) Szécsi, J.; Boson, B.; Johnsson, P.; Dupeyrot-Lacas, P.; Matrosovich, M.; Klenk, H.-D.; Klatzmann, D.; Volchkov, V.; Cosset, F.-L. Induction of Neutralising Antibodies by Virus-like Particles Harbouring Surface Proteins from Highly Pathogenic H5N1 and H7N1 Influenza Viruses. Virol. J. 2006, 3, 70.

(45) Schnitzbauer, J.; Strauss, M. T.; Schlichthaerle, T.; Schueder, F.; Jungmann, R. Super-Resolution Microscopy with DNA-PAINT. Nat. Protoc. 2017, 12 (6), 1198-1228.

(46) Pageon, S. V.; Nicovich, P. R.; Mollazade, M.; Tabarin, T.; Gaus, K. Clus-DoC: A Combined Cluster Detection and Colocalization Analysis for Single-Molecule Localization Microscopy Data. Mol. Biol. Cell 2016, 27 (22), 3627-3636.

(47) Anderson, E. J.; Rouphael, N. G.; Widge, A. T.; Jackson, L. A.; Roberts, P. C.; Makhene, M.; Chappell, J. D.; Denison, M. R.; Stevens, L. J.; Pruijssers, A. J.; McDermott, A. B.; Flach, B.; Lin, B. C.; Doria-Rose, N. A.; O’Dell, S.; Schmidt, S. D.; Corbett, K. S.; Swanson, P. A.; Padilla, M.; Neuzil, K. M.; Bennett, H.; Leav, B.; Makowski, M.; Albert, J.; Cross, K.; Edara, V. V.; Floyd, K.; Suthar, M. S.; Martinez, D. R.; Baric, R.; Buchanan, W.; Luke, C. J.; Phadke, V. K.; Rostad, C. A.; Ledgerwood, J. E.; Graham, B. S.; Beigel, J. H. Safety and Immunogenicity of SARS-CoV-2 MRNA-1273 Vaccine in Older Adults. N. Engl. J. Med. 2020, 383, 2427.

(48) Ura, T.; Yamashita, A.; Mizuki, N.; Okuda, K.; Shimada, M. New Vaccine Production Platforms Used in Developing SARS-CoV-2 Vaccine Candidates. Vaccine 2021, 39, 197.

(49) Sahin, U.; Muik, A.; Derhovanessian, E.; Vogler, I.; Kranz, L. M.; Vormehr, M.; Baum, A.; Pascal, K.; Quandt, J.; Maurus, D.; Brachtendorf, S.; Lörks, V.; Sikorski, J.; Hilker, R.; Becker, D.; Eller, A.-K.; Grützner, J.; Boesler, C.; Rosenbaum, C.; Kühnle, M.-C.; Luxemburger, U.; Kemmer-Brück, A.; Langer, D.; Bexon, M.; Bolte, S.; Karikó, K.; Palanche, T.; Fischer, B.; Schultz, A.; Shi, P.-Y.; FontesGarfias, C.; Perez, J. L.; Swanson, K. A.; Loschko, J.; Scully, I. L.; Cutler, M.; Kalina, W.; Kyratsous, C. A.; Cooper, D.; Dormitzer, P.
R.; Jansen, K. U.; Türeci, Ö. COVID-19 Vaccine BNT162b1 Elicits Human Antibody and T H 1 T Cell Responses. Nature 2020, 586 (7830), 594-599.

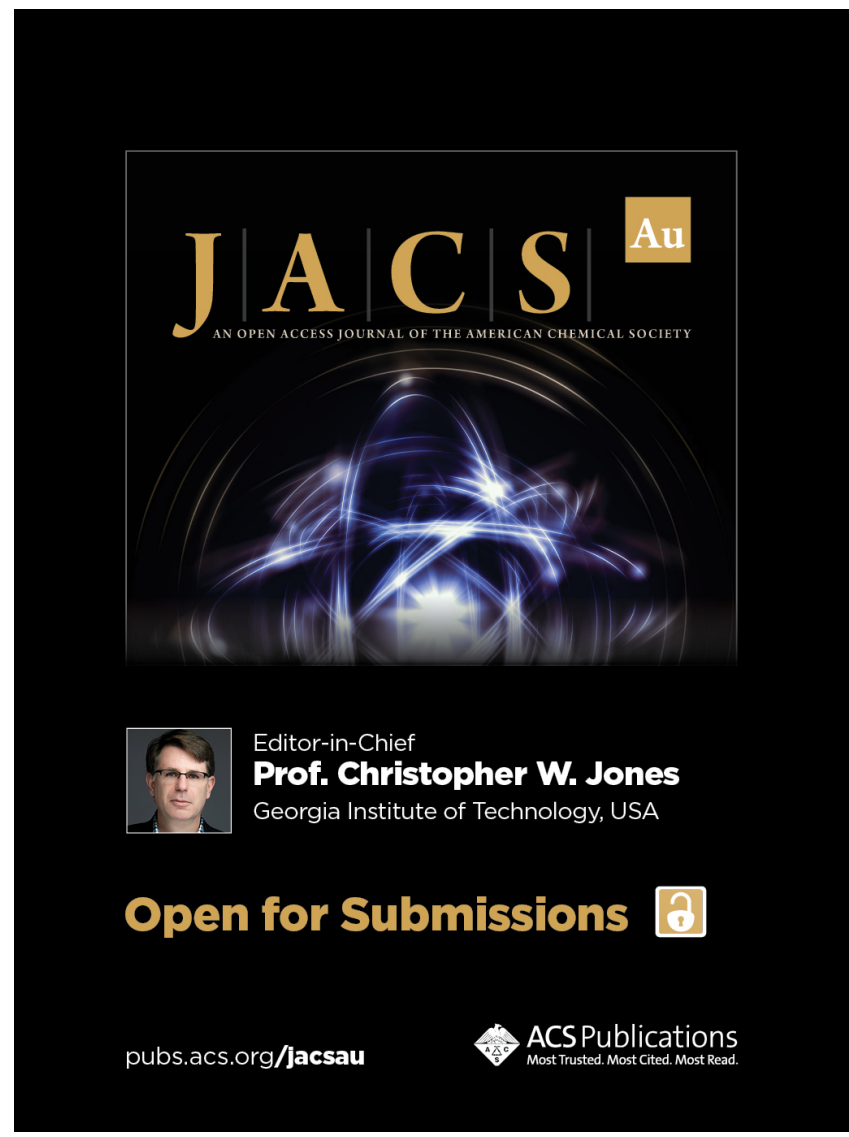

